\title{
La registrabilidad del olor en Colombia, una posibilidad marcaria para el siglo XXI ${ }^{1}$
}

\author{
The registrability of olfactory \\ signs in Colombia, a trademark \\ possibility for the 21st century
}

DOI: http://dx.doi.org/10.17981/juridcuc.13.1.2017.3

Artículo de Reflexión. Fecha de recepción: 18/07/2017 Fecha de aceptación: 14/09/2017

\author{
Camilo Andrés Díaz Trillos ${ }^{2}$ \\ Universidad del Rosario (Colombia) \\ camilod06gmail.com
}

Para citar este artículo:

Díaz, A. (2017). La registrabilidad del olor en Colombia, una posibilidad marcaria para el siglo XXI. JURÍDICAS CUC, vol. 13, no. 1, pp. 45-70. DOI: http://dx.doi.org/10.17981/ juridcuc.13.1.2017.3

\section{Resumen}

El presente artículo de reflexión busca determinar si es o no es posible registrar un signo olfativo de conformidad con lo dispuesto en la Decisión Andina 486 del 2000. Con base en la metodología de la dogmática analítica, se busca, en primer lugar, establecer cuáles son los criterios exigidos en la normativa andina para registrar un signo como marca.

\footnotetext{
${ }^{1}$ Artículo presentado como trabajo de grado para optar por el título de Magíster en Derecho con énfasis en Derecho Privado de la Universidad del Rosario (2017), producto de la investigación dirigida por el profesor Édgar Iván León Robayo. Lo anterior, dentro del proyecto de investigación Propiedad, Empresa y Consumo, desarrollado por la línea de investigación en Derecho Comercial del Grupo de Investigación en Derecho Privado de la Facultad de Jurisprudencia de la Universidad del Rosario.

${ }^{2}$ Abogado egresado de la Escuela de Derecho de la Universidad Sergio Arboleda. En la actualidad adelanta estudios de Maestría en Derecho con énfasis en Derecho Privado en la Universidad del Rosario.
}

- The author; licensee Universidad de la Costa - CUC. 
Una vez explicados los requisitos de registrabilidad, se estudiará si los signos formados por olores o aromas cumplen con estos requisitos; posteriormente, se hará un análisis en derecho comparado sobre las solicitudes y registros de marcas olfativas, y, finalmente, un estudio sobre las solicitudes de signos olfativos hechas en Colombia. En la práctica, la registrabilidad de los signos olfativos es incierta porque no existen criterios uniformes acerca de que un olor pueda cumplir con los requisitos de distintividad, percepción y representación gráfica exigidos en la Decisión Andina 486 de 2000.

\section{Palabras claves:}

Marca olfativa, requisitos de registrabilidad, distintividad, percepción, representación gráfica

\section{Abstract}

The present article of reflection, seeks to determine if it is or not possible to register an olfactory sign in accordance with the provisions of Andean Decision 486 of 2000 according to the methodology of analytical dogmatics, it is firstly sought to establish what are the criteria required In Andean legislation to register a sign as a trademark. Once the registration requirements have been explained, it will be examined whether the signs formed by odors or aromas comply with these requirements; Then a comparative law analysis will be done on the applications and registrations of olfactory marks and finally a study on the applications of olfactory signs made in Colombia. In practice, the registrability of olfactory signs is uncertain because there are no uniform criteria for an odor to meet the requirements of distinctiveness, perception and graphic representation required by Andean Decision 486 of 2000 .

Key words

Olfactory trademark, registration requirements, distinctiveness, perception, graphic representation 


\section{INTRODUCCIÓN}

En los mercados globalizados, por la gran cantidad de competidores que existen y la saturación del comercio de productos y servicios representados y publicitados por signos visuales, los empresarios para poder destacar sus productos o servicios se están valiendo de signos novedosos o no tradicionales con el fin de atraer y posicionar sus marcas entre los consumidores. Este dinamismo del mercado conlleva a la necesidad de los empresarios, no solo de usar, sino de proteger los signos no tradicionales como marcas con la finalidad de obtener derechos de explotación exclusiva sobre los signos novedosos y evitar que la competencia use signos similares o idénticos para atraer clientes.

Uno de los signos no tradicionales que tiene mayor impacto entre los consumidores es el olor. El olfato es el sentido encargado de captar y percibir los olores. Existe consenso entre los biólogos, neurólogos y psicólogos en considerar que el olfato es:

a. El más sensible de los sentidos, pues solo se requiere de unas pocas partículas químicas, emitidas por una sustancia olorosa, para excitar a una célula olfativa.

b. El sentido que genera mayor recordación en el ser humano. Según Sauri (2004) las personas recuerdan el 35\% de los olores que perciben; en cambio, su vista solo genera un $5 \%$ de recordación y su oído un $2 \%$.

El ser humano al percibir un olor, por ejemplo, un perfume, un alimento o una rosa, activa de modo automático su gran capacidad de asociación, y ésta, a su vez, se conecta con la memoria. He ahí la explicación del porqué los seres humanos, al percibir un olor, podemos evocar, con alto grado de detalle, lugares (restaurante donde percibimos por primera vez el olor de un alimento), personas (persona que usa el perfume cuyo olor estamos percibiendo), animales, productos, eventos (celebración del cumpleaños de la persona a la cual regalamos rosas cuyo olor es igual al que estamos percibiendo), etc. Según estudios que datan del 2014, el olfato humano es capaz de distinguir un billón de olores (López y Ramón, 2017). 
En este orden de ideas, cada día es más usual que los empresarios estén creando sus propios olores, aromas o fragancias, y que diseñen sus estrategias de publicidad de conformidad con el marketing olfativo ${ }^{3}$ para influenciar en la decisión de compra de sus potenciales clientes. Empresas como Walt Disney Company, Mario Hernández, Zara, Abercrombie o Coca-Cola Company utilizan olores determinados en sus productos y establecimientos de comercio.

En el ámbito jurídico, la protección de marcas ha recaído sobre los signos tradicionales, entendidos como aquellos conformados por letras, números, símbolos y figuras perceptibles visualmente, es decir, las marcas nominativas, figurativas y mixtas (Castro, 2012). Con la evolución de la tecnología y por las necesidades del mercado se ha planteado la necesidad de poder proteger los signos no tradicionales como son los olores.

La legislación andina respondió de manera positiva a esta necesidad manifiesta de los empresarios de proteger los signos olfativos. Prueba de ello es el artículo 134 de la Decisión Andina 486 de 2000, el cual brinda luz verde para que los olores sean considerados como marca: "Podrán constituir marcas, entre otros, los siguientes signos: c) los sonidos y los olores."

No obstante, existe una gran incertidumbre acerca de la posibilidad de poder registrar signos olfativos como marcas porque no hay un conceso de que el olor pueda cumplir con los requisitos de registrabilidad exigidos en el ordenamiento jurídico, por ejemplo, Fernández-Nóvoa (2004) afirma que los olores no son registrables.

En una investigación realizada por el Comité Permanente sobre el Derecho de Marcas, Diseños Industriales e Indicaciones Geográficas (SCT) sobre la registrabilidad de signos no tradicionales reveló que de las 72 oficinas de marcas que existen en el mundo, sólo 20 (27.7\%) permiten el registro de los signos olfativos (Organización Mundial de la Propiedad Intelectual [OMPI], 2006), lo cual demuestra que obtener la protección de un signo olfativo tiene muchas dificultades.

\footnotetext{
${ }^{3}$ Cleri (2016) expone "el nuevo emprendimiento propone utilizar el marketing olfativo para que actúe como diferencial identificativo, con la conciencia de que puede hacer más que el bombardeo de imágenes y sonidos". (p. 75).
} 
Teniendo en cuenta lo expuesto, el presente artículo se centrará en establecer si es posible registrar un signo olfativo de conformidad con lo dispuesto en la Decisión Andina 486 del 2000. La metodología que se utilizará para resolver este interrogante es la dogmática analítica; para ello, en primer lugar, se hará un estudio sobre los requisitos exigidos en la normativa andina para poder registrar un signo como marca. Una vez esbozados los requisitos de registrabilidad de las marcas, se abordará el tema de si los signos formados por olores o aromas cumplen con estos requisitos; posteriormente, se hará un análisis en derecho comparado sobre las solicitudes y registros de marcas olfativas; y finalmente, un estudio sobre las solicitudes de signos olfativos hechas en Colombia.

\section{Requisitos esenciales de las marcas}

La función principal de las marcas es la de identificar el producto y/o servicio de una empresa y, por consiguiente, distinguirlo de sus similares de la competencia. ${ }^{4}$ Para que un signo sea considerado como marca debe cumplir con los requisitos exigidos en la legislación vigente de cada país. El jurista y docente peruano Kresalija (2001), basado en el nivel de amplitud y flexibilidad de los requisitos legales que deben cumplir los signos para ser considerados marca, clasificó a los ordenamientos jurídicos en tres categorías, a saber:

a. Ordenamientos jurídicos amplios y flexibles, los cuales admiten cualquier signo con la única condición de ser suficientemente distintivo. Ejemplo de estos ordenamientos jurídicos son los de Estados Unidos y Canadá.

b. Ordenamientos jurídicos moderados, que establecen que un signo puede ser marca si es distintivo y es susceptible de representación gráfica; por ejemplo, el sistema jurídico chileno.

\footnotetext{
${ }^{4}$ Otamendi (1999) frente a la función principal de las marcas afirma: "La verdadera y única función esencial de la marca es distinguir un producto o servicio, de otros [...] La marca permite la distinción entre productos y servicios de una misma especie. Si el signo en cuestión no es apto para distinguir un producto o un servicio de otros, entonces no podrá ser marca en los términos señalados. Es fácil deducir que esta función distintiva es esencial” (p. 10-11).
} 
c. Ordenamientos jurídicos restrictivos, conforme a los cuales un signo es protegible como marca si es distintivo y perceptible únicamente por el sentido de la vista. Un ejemplo de este sistema era la Decisión 85 de la Comunidad Andina de 1971, la cual fue la primera norma andina en materia de propiedad industrial; en el artículo 56 establecía lo siguiente: "podrá registrarse como marcas de fábrica o de servicios, los signos que sean novedosos, visibles y suficientemente distintivos."

¿En cuál de las tres categorías de ordenamiento jurídico en referencia es factible registrar como marca signos olfativos? A nuestro entender, afirmamos que los signos olfativos no son registrables en la tercera categoría (ordenamientos jurídicos restrictivos), porque estos no se pueden percibir por medio del sentido de la vista.

Los signos olfativos encuentran mayores posibilidades de ser registrados como marcas en la primera categoría, esto es, ordenamientos jurídicos amplios y flexibles, puesto que estos no incorporan ningún impedimento de tipo legal para poder acceder al registro a los signos conformados por caracteres distintos a los visuales.

Nuestro ordenamiento jurídico actual es de corte moderado, ya que la Decisión Andina 486 de 2000, establece que un signo puede ser protegido como marca siempre que cumpla con los siguientes requisitos:

a.Ser distintivo.

b. Ser perceptible.

c. Ser susceptible de representarse gráficamente.

\section{Requisito de distintividad}

El primer requisito exigido por la normativa andina para el registro de marcas es que el signo "sea apto para distinguir productos o servicios en el mercado". (Decisión 486 de la Comunidad Andina de Naciones, 2000, art. 134). Esto es conocido como el carácter distintivo de la marca. El profesor Cárdenas (2003) define la distintividad, en forma general, como: 
Lo distintivo es lo que hace que una persona, cosa $u$ objeto, por sus características subjetivas u objetivas o por rasgos, naturaleza, funciones o condiciones interiores o exteriores sean distinta de la otra. Y lo distintivo, por lo tanto, conduce a diferenciar dos personas u objetos (pp.195-196).

En materia marcaria (basados en una interpretación literal de la norma andina), la distintividad consiste en que el signo debe ser adecuado para diferenciar productos y servicios dentro del mercado.

El doctrinante Parra (2001), en un análisis más profundo expone que la distintividad tiene dos vertientes, a saber:

a.La distintividad en sentido estricto, $\mathrm{y}$

b.la distintividad en sentido amplio.

La distintividad en sentido estricto se circunscribe a la función esencial de la marca de poder distinguir o individualizar bienes y/o servicios con la finalidad de que el público consumidor los identifique. Leamos lo que al respecto escribió el doctor Pachón (Pachón y Sánchez, 1995), consagrado estudioso de la propiedad industrial y el derecho de autor: "El requisito fundamental que debe reunir el signo para poder registrarse como marca es que pueda cumplir con la función de distinguir los productos o servicios" (p.197).

Por su parte, la distintividad en sentido amplio está ligada estrechamente con las causales de irregistrabilidad consagradas en el artículo 135, literales c, d, e, f y g, y el artículo 136 de la Decisión Andina 486 de 2000. Basado en estos artículos, Parra (2001) afirma que:

La falta de distintividad relativa se presenta generalmente unida al principio de especialidad y debe atarse siempre a la clase de productos o servicios respecto de la cual se pretende registrar la marca y, por ende, respecto de la cual se deba hacer el análisis de registrabilidad, determinando si con ellos se puede generar confusión entre el público o se puede ir en contra del principio de la libre competencia al monopolizar un signo que es necesario para desarrollar determinada actividad empresarial, siendo en consecuencia estos conceptos, los que se deben tener en cuenta para conocer cuando estamos ante un signo que adolezca de falta de distintividad relativa (p. 36). 
El profesor Metke (2006), al interpretar el primer párrafo del artículo 134 de la Decisión 486 de 2000, se refiere a la aptitud marcaria:

La aptitud marcaria comprende aspectos tales como la estructura de la marca, la cual no puede ser de una simplicidad tan elemental que no permita asociarla con un signo marcario [...]; tampoco puede ser de una estructura demasiado compleja [...]. Tampoco sería apto como marca el signo que representa un valor intrínseco que trascienda el propio de un signo distintivo [...]. Bajo este criterio de la aptitud marcaria también podría encuadrarse el caso del signo que esté constituido por un atributo del producto (pp. 27-29).

Como vemos, el profesor Metke (2006) relaciona el requisito de distintividad, con la capacidad de la marca de diferenciar el producto o servicio. En este aspecto, tanto la doctrina ${ }^{5}$ como la jurisprudencia ${ }^{6}$ establecen dos tipos de distintividad: la intrínseca y la extrínseca.

La distintividad intrínseca se refiere a la capacidad del signo de identificar productos y/o servicios, es decir, de que los productos o servicios que pretende identificar el signo no sean descriptivos, genéricos, usuales o engañosos. La distintividad extrínseca se refiere a que el signo no sea similar o idéntico a signos distintivos registrados o solicitados prioritariamente por terceros.

En nuestra legislación colombiana los signos que carecen de distintividad intrínseca son causales absolutas de irregistrabilidad, reguladas en el artículo 135 de la Decisión Andina 486 de 2000. En consecuencia, un tercero o la oficina de marcas pueden presentar en cualquier tiempo la acción de nulidad. Por otro lado, los signos que carecen de distintividad extrínseca son causales de irregistrabilidad relativas, reguladas en

\footnotetext{
${ }^{5}$ Doctrina expresada por autores como Rodolfo Montoya Lizarazu, Juan David Castro García, Ricardo Metke Méndez.

${ }^{6}$ El Tribunal de Justicia de la Comunidad Andina. Proceso 26-IP-2012, del 18 de abril de 2012, sobre la distintividad expresa lo siguiente: "Es importante advertir que la distintividad tiene un doble aspecto: 1) distintividad intrínseca, mediante la cual se determina la capacidad que debe tener el signo para distinguir productos o servicios en el mercado; y, 2) distintividad extrínseca, mediante la cual se determina la capacidad del signo de diferenciarse de otros signos en el mercado."
} 
el artículo 136. Por tanto, sólo los que tengan un interés real pueden presentar la acción de nulidad dentro de los cinco años siguientes, conforme con lo establecido en el artículo 172 de la Decisión 486.

El uso del signo en el mercado puede tener dos efectos respecto a la distintividad, a saber:

a.La vulgarización de la marca

b. La distintividad sobrevenida

La vulgarización de marca hace referencia a las marcas registradas que, por el gran reconocimiento adquirido de parte de los competidores y de los consumidores, pierde su fuerza distintiva porque el producto o servicio que identifica la marca se confunde con ésta; por ejemplo, el icopor era una marca comercial que identificaba al poliestireno expandido, un material plástico y rígido que se usa en la elaboración de envases y en la industria de la construcción. Debido al reconocimiento adquirido, los consumidores se referían al poliestireno expandido como icopor. Esto trajo como consecuencia que la marca (Icopor) perdiera su fuerza distintiva. Esto lleva a preguntarse: ¿qué prevé la legislación referente a la vulgarización de una marca?

La legislación andina en el artículo 169 de la Decisión 486 de 2000 establece que cuando una marca se vulgariza, la oficina de marcas o un tercero pueden solicitar que se cancele el registro por haber perdido la distintividad.

El otro efecto respecto a la distintividad que puede generar el uso del signo en el mercado es el de la distintividad sobrevenida, fenómeno conocido también como la teoría del segundo significado (secondary meaning). La naturaleza de este efecto es exactamente contraria a la de la vulgarización de la marca, pues consiste en un signo que no ha sido registrado como marca debido a que carece de distintividad, sin embargo, en virtud de su uso constante o reiterado en el mercado, los consumidores reconocen el origen empresarial de dicho signo, en consecuencia, éste adquiere la suficiente distintividad para poder ser registrado. La Decisión Andina 486 de 2000, en el inciso final del artículo 135, admite la figura de la distintividad sobrevenida y la regula. 
A modo de síntesis, se presenta la siguiente tabla sobre las diferencias entre los dos efectos respecto a la distintividad generados por el uso del signo en el mercado.

Tabla 1.

Diferencias en los efectos de la distintividad de la marca.

\begin{tabular}{ll}
\hline \multicolumn{1}{c}{$\begin{array}{c}\text { VULGARIZACIÓN } \\
\text { DE LA MARCA }\end{array}$} & \multicolumn{1}{c}{$\begin{array}{c}\text { DISTINTIVIDAD } \\
\text { SOBREVENIDA }\end{array}$} \\
\hline Aplica para marcas registradas & $\begin{array}{l}\text { Aplica para signos no registrados } \\
\text { como marca. }\end{array}$ \\
$\begin{array}{l}\text { La marca pierde su carácter dis- } \\
\text { tintivo porque el producto o servi- } \\
\text { cio que representa se confunde con } \\
\text { ella. }\end{array}$ & $\begin{array}{l}\text { virtud de que los consumidores reco- } \\
\text { nocen el origen empresarial del pro- } \\
\text { ducto o servicio que él representa. }\end{array}$ \\
$\begin{array}{l}\text { El registro de la marca puede ser } \\
\text { cancelado debido a la pérdida de la } \\
\text { distintividad. }\end{array}$ & $\begin{array}{l}\text { El signo puede ser registrado en vir- } \\
\text { tud de la distintividad adquirida. }\end{array}$ \\
\hline
\end{tabular}

\section{Requisito de perceptibilidad}

Este requisito no está expuesto de forma textual en la normativa andina, pero se entiende incluido dentro de los requisitos esenciales que debe tener una marca. El requisito de perceptibilidad es imprescindible debido a que la marca es un intangible (Martínez y Vargas, 2015).

El Tribunal de Justicia de la Comunidad Andina de Naciones, en el proceso 240-IP-2013, con el Magistrado ponente Luis José Diez Canseco Núñez, el 20 de febrero de 2014 sobre la perceptibilidad marcaria, ha expresado lo siguiente:

El requisito de perceptibilidad, que estaba expresamente establecido en la Decisión 344, está implícitamente contenido en la definición del artículo 134 mencionado, toda vez un signo para que pueda ser captado y apreciado, es necesario que pase a ser una expresión material identificable, a fin de que al ser aprehendida por medios sensoriales y asimilada por la inteligencia, penetre en la memoria de los consumidores o usuarios. 
La perceptibilidad permite que existan signos táctiles, sonoros, olfativos, gustativos y visuales. Sin embargo, la perceptibilidad marcaria no se debe entender únicamente como la facultad de que el signo sea captado por los sentidos, sino que sea percibido. Es decir, que con base en los datos suministrados por los sentidos y la activación inmediata de la operación sicológica llamada percepción sensorial, la mente o inteligencia de los individuos identifique plenamente el signo, esto es, reconozca la procedencia empresarial del producto o servicio que representa el signo en referencia. ${ }^{7}$

A modo de síntesis, decimos que la perceptibilidad requiere del concurso de dos operaciones muy relacionadas: una de tipo fisiológico (sensación) y otra de tipo sicológico (percepción). La sensación recoge o capta información del ambiente (colores, texturas, olores, etc.) y la envía al cerebro. La sensación es catalogada como una operación fisiológica por cuanto en ella los protagonistas son los órganos de los sentidos. A su vez, la percepción realiza las funciones de seleccionar, organizar e interpretar las sensaciones. Estas funciones las realiza con base en las experiencias y recuerdos previos, esto significa que sus protagonistas son el cerebro y la memoria. A decir de los sicólogos cognitivos la percepción se apoya en las sensaciones y genera "una unidad cognitiva de nivel superior” (De Vega, 2001, p. 369).

\section{Requisito de representación gráfica}

El Comité Permanente sobre el Derecho de Marcas, Diseños Industriales e Indicaciones Geográficas (SCT), en la decimoséptima sesión (mayo 7 al 11 de 2007), en un estudio realizado en 73 oficinas de marcas, afirmó que para poner en marcha el proceso de registro de una marca es necesario representarla, y que el método más aceptado para este fin es la representación gráfica.

\footnotetext{
${ }^{7}$ El profesor Arias (2015) expresa lo siguiente: “En mi opinión, el requisito de perceptibilidad está íntimamente relacionado con la capacidad distintiva intrínseca del signo, por lo que no basta que el signo sea meramente percibido por los sentidos, sino que, además, dicha percepción debe ser capaz de ser asimilada por la inteligencia, de forma que le permita al consumidor identificar el producto o servicio marcado con dicho signo" (p. 90).
} 
En la legislación colombiana, para que un signo sea considerado marca, se requiere que éste sea susceptible de representarse gráficamente. ¿Cuál es la razón de ser del requisito de representación gráfica? Sencillamente, la representación gráfica se exige o requiere con la finalidad de que el signo a registrar sea percibido por los consumidores.

¿En qué consiste la representación gráfica de un signo a registrar como marca? Según el Tribunal de Justicia de la Comunidad Andina, Proceso 08-IP-2013, la representación gráfica consiste en:

La posibilidad de que el signo a registrar como marca sea descrito mediante palabras, gráficos, signos, colores, figuras, etc., de tal manera que sus componentes puedan ser apreciados por quien lo observe. Esta característica es importante para la publicación de las solicitudes de registro en los medios oficiales.

La representación gráfica de los signos tiene las siguientes finalidades:

a. Posibilitar y facilitar a la oficina de marcas la realización de los estudios de registrabilidad y publicación de la marca.

b. Brindar a los terceros la información clara y suficiente para determinar si la solicitud de registro de un signo específico afecta o no sus derechos en razón de ser o no ser, respectivamente, confundible con sus signos.

c. Posibilitar a los consumidores la percepción de la marca. ${ }^{8}$

\footnotetext{
${ }^{8}$ Fernández-Nóvoa, (2004) refiriéndose a las funciones de la representación gráfica, afirma que: "Es indudable que, en la fase inicial de la tramitación, la Oficina de Marcas no podría realizar las pertinentes publicaciones y proceder a la aplicación de las prohibiciones de registro si la solicitud de la marca no contuviese una representación gráfica adecuada del signo. En esta fase inicial, los titulares de marcas anteriores tampoco podrían entablar oposiciones si las publicaciones de la Oficina de Marcas no suministrasen una información suficiente acerca de la representación gráfica del signo solicitado como marca. Una vez registrada la marca, y a lo largo de toda su vida, el requisito de representación gráfica sigue siendo fundamental. Piénsese en efecto, que la hipótesis de colisión entre marcas registradas en el marco de procedimiento de infracción, el Tribunal debe tener a su disposición una información clara y suficiente acerca de los signos en pugna, información que debe ser proporcionada por las publicaciones oficiales en las que los correspondientes signos son representados gráficamente" (p. 43).
} 
La representación gráfica es uno de los mayores obstáculos para las marcas no tradicionales no visuales, pues presupone que para que un signo sea considerado marca no basta con que éste sea lo suficientemente distintivo y que sea captado por alguno de los sentidos, sino que el signo sea susceptible de ser representado y captado por medios visuales.

Delimitados los conceptos y requisitos esenciales que deben cumplir cualquier signo para ser registrado como marca bajo la normativa andina, a continuación, se hará un análisis sobre estos requisitos aplicados a los signos olfativos.

\section{La distintividad de las marcas olfativas}

El primer requisito que deben cumplir los signos olfativos para ser protegidos como marcas es determinar si el olor tiene la capacidad de identificar productos y/o servicios en el mercado. No existe consenso entre los expertos en propiedad industrial acerca del grado de distintividad de los olores. El debate referente a este tema se centra en los siguientes puntos:

a.Determinar si el olor por sí solo puede identificar productos y/o servicios en el mercado.

b.El análisis de confundibilidad entre los olores.

En el contexto del debate acerca del primer punto en mención, la doctrina especializada ha establecido una diferenciación entre:

- Olores primarios (primary scents)

- Olores de productos (product scents)

- Olores únicos (unique scents)

Los llamados olores primarios se refieren a los productos cuya finalidad es emitir un olor o una fragancia; ejemplo: perfumes y ambientadores. Los olores de productos se refieren a aquellos productos que usualmente se les incorpora un aroma, sin que este olor sea su característica primordial, por ejemplo, los jabones y cosméticos. Los olores únicos son aquellos olores incorporados a productos que no producen un olor característico (Hernández, 2008).

Los olores primarios por sí solos no tendrían fuerza distintiva suficiente para identificar productos porque el olor es una 
característica o atributo inherente de la naturaleza del producto. La oficina de marcas del Reino Unido, atendiendo esta teoría, rechazó el registro de la marca olfativa del perfume No. 5 solicitada por Chanel S.A., argumentando que el olor es la esencia del producto (Castonguay, 2009).

Los olores de productos tampoco tienen fuerza distintiva porque al ser aromas habitualmente utilizados en los productos, el público consumidor al percibir el olor no podría asociarlo con un origen empresarial determinado por ser un aroma comúnmente utilizado en el mercado.

Los olores únicos podrían tener fuerza distintiva, ya que el olor que le impregnan al producto o servicio es arbitrario; en consecuencia, al no ser derivado del bien ni ser usado de forma habitual por los comerciantes, el consumidor al sentir el aroma puede identificar el producto o servicio y atribuirle un origen empresarial determinado. En efecto, las pocas marcas olfativas registradas, son las de olor único, por ejemplo, en Estados Unidos el USPTO aceptó como registro el "olor de cereza para hilos de costura" (Castro, 2012, p. 312).

En la legislación andina, el olor que se pretenda solicitar como marca debe ser apto para identificar productos o servicios y no debe ser genérico, descriptivo, funcional o usual. En este orden de ideas, en principio los signos conformados por primary scents o product scents no podrían ser registrados como marca bajo la legislación andina, debido a que estarían inmersos en las causales de irregistrabilidad del artículo 135 de la Decisión Andina 486 de 2000, ya que los signos conformados por este tipo de olores carecerían de aptitud distintiva o de distintividad intrínseca.

Adicional a lo anterior, frente a la distintividad de los olores o aromas, la doctrina ha discutido si el olor tiene fuerza distintiva inherente, es decir, si el olor por sí solo tiene la capacidad de identificar productos y servicios en el mercado, o si es necesario que el olor adquiera fuerza distintiva mediante el uso previo en el mercado (secondary meaning) (Kresalija, 2001).

La Oficina de Armonización del Mercado Interior (OAMI), en el año 1999, cuando concedió la marca olfativa "olor a hierba recién cortada" para identificar pelotas de tenis, consideró que 
este olor era lo suficientemente distintivo. Este fallo ha recibido diversas críticas, por ejemplo, la profesora Llogrebat (2007), en referencia a dicho fallo, anotó que:

Se trata de supuestos aislados y este caso, a mi juicio, merece una crítica rigurosa [...] el solicitante no probó que su marca olfativa hubiera alcanzado el secondary meaning (distintividad sobrevenida), es decir, que los consumidores hayan llegado a distinguir el origen empresarial por el olor a hierba cortada de las pelotas de tenis (p. 67).

En Estados Unidos se ha establecido que una marca olfativa puede ser registrable siempre y cuando el signo haya adquirido un segundo significado, es decir, que el olor haya sido utilizado anteriormente por el solicitante y que los consumidores al percibir el olor reconozcan el origen empresarial (Escoffie y Jin, 2003).

El segundo gran debate se centra en la distintividad extrínseca de los olores, en especial sobre el examen de confusión entre signos olfativos. Un sector de la doctrina afirma que el sentido del olfato de los consumidores promedios tiene una limitada capacidad para diferenciar y distinguir olores precisos, además, las fragancias o aromas que pueden ser usados dentro del comercio son muy limitadas y son altamente similares, lo cual genera que el examen de confusión entre un signo olfativo registrado con uno solicitado sea muy difícil de aplicar (Kresalija, 2001).

Contrario a lo anterior, exponer que el olfato humano no tiene la capacidad suficiente para identificar aromas determinados, es negar la fuerza de percepción que tiene el sentido del olfato. Si bien es cierto que el análisis de confusión es más fácil hacerlo entre signos visibles, el consumidor puede identificar productos o servicios mediante el olor, y podrá determinar cuando se solicite o se use una fragancia similar o idéntica a una registrada (Kresalija, 2001), más aun si se trata de unique scents porque el consumidor al sentir el aroma inmediatamente lo asocia con el producto registrado, y al momento de que un tercero use un aroma similar para identificar el mismo producto, evidentemente existirá riesgo de confusión. 
De forma simple, los signos olfativos cumplen con el requisito de perceptibilidad porque el ser humano puede captar el signo mediante el sentido del olfato. No obstante, al hacer un análisis profundo sobre el requisito de perceptibilidad, Hernández (2008) expresa que no basta únicamente con que el consumidor pueda percibir el aroma por medio del olfato, si no que una vez que el consumidor perciba el olor este pueda identificar el producto o servicio y asociarlo a un origen empresarial determinado. En el evento en que el consumidor, al percibir el olor, no lo asocie con el producto, sino como una característica secundaria u ornamental, el signo no sería registrable porque no solo carecería de distintividad, sino que el signo no sería perceptible como marca.

\section{Representación gráfica de los olores}

El obstáculo más grande sobre la registrabilidad de la marca olfativa es el requisito de representación gráfica debido a la dificultad de expresar un olor mediante palabras, símbolos, números o gráficos.

En una célebre interpretación del Tribunal de Justicia de la Comunidades Europeas (TJCE), sobre la representación gráfi$\mathrm{ca}^{9}$, conocida como el caso Sieckmann, el solicitante expuso las siguientes formas para representar gráficamente el olor:

- Fórmula química del compuesto del olor

- Descripción del olor por medio de palabras

- Una muestra del olor

- La combinación de todos los anteriores

El TJCE, para el asunto C-273/00, caso Ralf Sieckmann/ Deutches Patent-und Markenamt, rechazó todas las formas expuestas por el solicitante por las siguientes razones:

\footnotetext{
9 La Unión Europa, en la Directiva 89/104/CEE, la cual regula el régimen de marcas comunitarias, exige de forma taxativa que un signo para ser considerado como marca debe poder representarse gráficamente en el artículo 2, que dice lo siguiente: "Podrán constituir marcas todos los signos que puedan ser objeto de una representación gráfica, especialmente las palabras, incluidos los nombres de personas, los dibujos, las letras, las cifras, la forma del producto o de su presentación, a condición de que tales signos sean apropiados para distinguir los productos o los servicios de una empresa de los de otras."
} 
- La fórmula química no describe el olor de la sustancia, sino que describe a la misma sustancia. Adicionalmente, la fórmula química no es clara ni precisa y muy pocas personas podrían interpretar una fragancia a partir de la fórmula química.

- La descripción del olor por medio de palabras, si bien en principio podría cumplir con el requisito de representación gráfica, no cumple con las exigencias de claridad y precisión porque la descripción del olor es subjetiva.

- El depósito de una muestra del olor, no equivale a representar gráficamente el signo. Además, en el evento de que se aceptara una muestra del olor, ésta no resultaría precisa ni clara porque el olor varía por los cambios de ambiente, temperatura y tiempo, por lo tanto, no es estable ni duradera.

- La combinación de las tres modalidades anteriores tampoco sería aceptable porque enviaría distintas señales sobre el signo, lo cual generaría mayor incertidumbre entre los receptores.

En consecuencia, el Tribunal concluyó que la representación gráfica debe ser "clara, precisa, completa en sí misma, fácilmente accesible, inteligible, duradera y objetiva”. En armonía con la doctrina generada por este fallo, se puede interpretar que en las legislaciones donde se exija la representación gráfica en los signos no se puede acceder al registro de los olores como marca (Fernández-Nóvoa, 2004).

Siguiendo los lineamientos establecidos por el TJCE, algunos autores consideran que en el estado actual no es posible representar gráficamente un olor, pero en un futuro esto será posible $^{10}$ a causa de los grandes avances tecnológicos, como, por ejemplo, la creación de medios digitales que permitan capturar el olor de tal forma que su representación gráfica sea clara, precisa, completa en sí misma, fácilmente accesible, inteligible, duradera y objetiva.

${ }^{10}$ Ver más (Kresalija, p. 171). 
En contravía de la idea descrita en el párrafo anterior, otros autores como Montoya (2014) afirman que con los métodos actuales es posible representar gráficamente un olor, más exactamente, por medio del análisis cromatográfico en la fase gaseosa de la sustancia que produce el olor. Esta técnica consiste en separar todos los compuestos de un olor con la finalidad de poder caracterizarlos (Díaz y Pérez, 2006).

$\mathrm{Al}$ analizar las marcas olfativas que se han registrado en el mundo, se pone en evidencia que la representación gráfica del olor que ha sido aceptada es la descripción del aroma por medio de palabras. Por ejemplo, la oficina de marcas del Reino Unido aceptó el registro de la marca olfativa el "olor floral que recuerda al de las rosas" para identificar neumáticos, y el "olor acre de la cerveza amarga" para identificar dardos.

\section{Marcas olfativas registradas en el mundo}

En el ámbito internacional existen países cuyas legislaciones permiten la registrabilidad de los signos olfativos; ejemplo: Estados Unidos y Argentina. La legislación de Estados Unidos tiene establecido los siguientes criterios para obtener el registro de un signo olfativo:

a. Que el solicitante sea la única persona que comercializa los bienes afectados;

b. Que la fragancia no sea un atributo inherente o una característica natural de los bienes, sino que debe ser una característica aportada por el solicitante;

c. El solicitante debe haber puesto de relieve y dado a conocer la marca olfativa en su publicidad; y

d. El solicitante debe demostrar que los clientes, comerciantes y distribuidores de sus productos reconocen a su empresa como origen de dichos bienes (OMPI, 2006, pp. 10-9).

Frente a lo anterior, si bien el registro de un signo olfativo es posible, se deben cumplir estas condiciones y esto limita las probabilidades de proteger como marca un signo olfativo.

En Argentina, a la empresa L'Oréal se le concedieron una serie de marcas olfativas constituidas de fragancia de diferentes frutas para identificar envases de productos de la clase 03 
internacional. El Tribunal que conoció sobre las oposiciones expuso que en la legislación argentina no existe ningún requisito esencial que exija que los signos olfativos deban ser perceptibles mediante el sentido de la vista o deba ser representadas gráficamente, en consecuencia, al enviarlo al IMPI éstas fueron concedidas (Martin y Zuccherino, 2010).

$\mathrm{Al}$ analizar estas dos legislaciones, es menester mencionar que, frente a la fuerza distintiva de los olores, aplican métodos distintos; en Estados Unidos, para acceder al registro de una marca el olor, debe haber adquirido una distintividad sobrevenida, mientras que, en Argentina, el olor no debe estar incurso en las causales de irregistrabilidad consagradas en la ley.

En el derecho internacional, en las legislaciones donde no se exige la representación gráfica del signo olfativo es más factible que la oficina de marca acepte su registro como marca. No obstante, todas las marcas olfativas registradas han tenido una representación gráfica visual consistente en la descripción por medio de lenguaje escrito del aroma que se pretende registrar.

Algunos sectores de la doctrina han argumentado que el requisito de representación gráfica es innecesario y que los signos solicitados ante las oficinas de marcas pueden utilizar medios alternativos para dar a conocer o entender el signo solicitado como marca (Balañá, 2006). Frente a este tipo de solicitudes, algunas legislaciones han eliminado el requisito de representación gráfica necesario para que un signo sea protegido como marca. La Oficina de Propiedad Intelectual de la Unión Europea (EUIPO), mediante el reglamento (UE) 2015/2424 del Parlamento Europeo y del Consejo del 16 de diciembre de 2015, modificó el reglamento (CE) No. 207/2009 del Consejo sobre la marca comunitaria, pues eliminó taxativamente el requisito de representación gráfica. En su reemplazo estableció lo siguiente:

Artículo 4. Podrán constituir marcas de la Unión cualesquiera signos, en particular, las palabras, incluidos los nombres de personas, los dibujos, las letras, las cifras, los colores, la forma del producto o de su embalaje, o los sonidos, con la condición de que tales signos sean apropiados para: 
[...] ser representados en el Registro de Marcas de la Unión Europea (el "Registro") de manera que permita a las autoridades competentes y al público en general determinar el objeto claro y preciso de la protección otorgada a su titular.

En un análisis exegético, se podría concluir que este cambio legislativo si bien constituye un avance a la registrabilidad de marcas no visibles como lo son los signos olfativos, la redacción del primer parágrafo del artículo de forma taxativa permite el registro de marcas nominativas, figurativas, mixtas, de color, las tridimensionales, los tradedress y las sonoras.

No obstante, la interpretación de este artículo debe ser amplia con la finalidad que cualquier signo que sea solicitado debe ser representado por un método que permita conocer el signo de forma clara y precisa, lo cual facilitaría el registro de signos olfativos, pero debe tenerse en cuenta que el método utilizado debe ser claro y preciso.

\section{Marcas olfativas solicitadas en Colombia}

En el caso colombiano, todas las marcas olfativas solicitadas han sido negadas por la Superintendencia de Industria y Comercio (SIC) por no cumplir con el requisito de representación gráfica.

La primera solicitud en Colombia para el registro de un signo olfativo fue presentada por Laboratorios Cero S.A. y tramitada bajo el expediente no. 05047635. El solicitante pretendía obtener el registro de la anterior marca olfativa por medio de la descripción del olor junto con el resultado de un examen cromatográfico para identificar productos correspondientes a la clase 3 Internacional de Niza. ${ }^{11}$

\footnotetext{
${ }^{11}$ Los productos que se pretendían proteger son los siguientes: "cremas antipañaliticas no medicadas, geles, champús, vaselinas, aceites corporales, protectores labiales, removedores, talcos, así como preparaciones para blanquear y otras sustancias para la colada; preparaciones para limpiar, pulir, desengrasar y raspas; (preparaciones abrasivas) jabones; perfumería, aceites esenciales, cosméticos, lociones para el cabello; dentífricos.”
} 


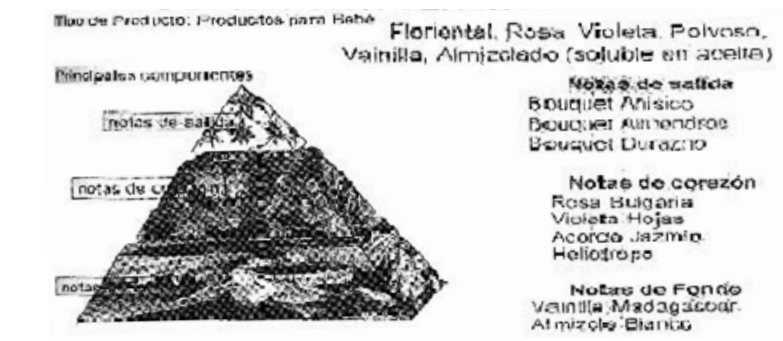

Figura 1. Cromatograma. Descripción olfativa.

Fuente: República de Colombia. Superintendencia de Industria y Comercio (2005)

La SIC mediante la resolución 17152 del 4 de abril de 2009, negó en segunda instancia la solicitud de registro del signo olfativo por las siguientes razones:

a. Siguiendo los lineamientos del caso Sieckmann, el examinador consideró que la representación gráfica del olor no era posible, y que las técnicas especializadas para identificar olores, como estudios cromatográficos u otras, no eran comprensibles para el común de las personas, en consecuencia, la representación gráfica no resultaba completa ni fácilmente accesible e inteligible.

b. Los productos de la clase 3 Internacional de Niza que se pretendían proteger por el solicitante de la marca, por lo general están dotados de olores, por lo tanto, el aroma incorporado a los productos fue entendido como una característica del producto. Con base en estos hechos, el examinador determinó que el olor de este tipo de productos carecía de distintividad intrínseca.

La segunda solicitud de un signo olfativa fue realizada por Empresas Públicas de Medellín E.S.P., el cual se tramitó con el No. de expediente 10039538. El signo era el siguiente:

Olor compuesto por (a) un fondo predominante de carácter cítrico, integrado con (b) un toque destacado de aroma a jazmín y, (c) de manera complementaria, notas ligeras consistentes en mezclas de: jazmín con limón, eucalipto con menta y limoncillo. Evoca un ambiente campestre relacionado con lugares cercanos a fuentes de agua natural; igualmente, brinda recordación sobre elementos de naturaleza pura y viva, generando sensaciones de vitalidad, confort, tranquilidad y frescura para identificar servicios de la clase 35 Internacional de Niza. 
La SIC al hacer el examen de registrabilidad al signo, negó el registro por medio de la resolución 90525 del 29 de diciembre de 2016. La SIC argumentó lo siguiente:

a. En primer lugar, al igual que en la resolución 17152 estableció las condiciones expuestas en el caso Sieckmann para representar gráficamente un olor.

b. La descripción escrita del olor, no cumple con el requisito de representación gráfica porque la descripción realizada es ambigua, ya que no es posible determinar con plena seguridad los aromas descritos, además, la descripción es subjetiva debido a que cada persona puede percibir el olor de diferente manera, en consecuencia, la descripción del olor no es precisa, clara, fácilmente inteligible ni objetiva.

Con base en lo anterior, los signos olfativos no son registrables en Colombia por la imposibilidad de representar gráficamente el olor, además, se debe tener en cuenta que el aroma que se pretenda registrar no sea una característica del producto o servicio. Aunque es preciso mencionar que el requisito de representación gráfica en el ordenamiento andino está adaptándose a las nuevas marcas permitiendo que signos atípicos se representen gráficamente por medios alternativos a los visuales. El TJCA, en una de las sentencias más importante de los últimos años, en consulta hecha por la SIC acerca de los requisitos de representación gráfica y distintividad de los signos táctiles, proceso 242IP-2015, con M.P. Luis José Diez Canseco Núñez, el 24 de agosto de 2015, frente a la representación gráfica, afirma lo siguiente:

Este Tribunal considera que la descripción simple ayuda al examinador a determinar lo que se pretende registrar, por cuanto la inspección visual de la marca generalmente no sirve para brindar precisión o certeza en el caso de signos no visibles. La descripción simple también permite darse cuenta de características de la marca que no aparecen en dibujos o gráficos que representan la marca. Esto último resulta fundamental en el caso particular que nos ocupa, por cuanto ciertos tipos de textura son casi imposibles de recrear mediante simples dibujos o gráficos. Por lo tanto, debe tomarse en cuenta la posibilidad de exigir, y luego publicar, de manera suplementaria o adicional a la representación técnica principal, una descripción simple de la marca solicitada, para así otorgar una mayor comprensión y precisión de lo reivindicado mediante la solicitud de registro. 
En consecuencia, este Tribunal interpreta de una manera amplia lo que se entiende por representación gráfica, siendo necesaria la concurrencia de dos requisitos: i) la descripción clara, precisa y completa del signo, incluyendo un dibujo tridimensional o fotografía; $y$, ii) muestra física de la marca táctil. Ambos requisitos tienen como objetivo representar de una manera suficiente el signo, tomando en especial consideración el principio de precisión desarrollado en la presente ponencia.

Frente a esta sentencia, en la legislación andina se pueden utilizar medios distintos a los gráficos para representar un signo no visible, esto amplía las posibilidades de poder representar un signo olfativo.

¿Es posible registrar signos olfativos en Colombia? En nuestro criterio para poder obtener la protección de un signo olfativo en Colombia, éste debe cumplir con los siguientes parámetros:

1. Las características principales, secundarias o funcionalidades de los productos o servicios que se pretendan identificar mediante el signo olfativo no deben tener ninguna relación directa o indirecta con el olor que se aspira a registrar.

2. Demostrar que los consumidores, al percibir el olor que se pretenda registrar, pueden identificar el origen empresarial de los productos o servicios identificados por el olor.

3. Para representar gráficamente el signo olfativo pueden utilizarse varios métodos, por ejemplo, mediante una descripción sencilla y comprensible del olor u olores que componen el signo (cuyo origen empresarial ya es identificado por los consumidores). A esta descripción se le suma una prueba técnica que corrobore el o los olores que se están describiendo a fin de evitar la subjetividad. De este modo, se cumpliría con las exigencias legales establecidas en la Decisión Andina 486 de 2000.

Es importante resaltar que ningún signo olfativo solicitado en la legislación andina ha sido estudiado por el TJCA, por lo tanto, no existe un pronunciamiento jurisprudencial que permita establecer con certeza si en el marco del derecho andino es posible o no registrar un signo olfativo. 


\section{CONCLUSIONES}

La registrabilidad de los signos olfativos en Colombia presenta múltiples dificultades debido a que no existe un criterio uniforme en la doctrina ni en la jurisprudencia acerca de que un olor pueda cumplir con los requisitos de distintividad, percepción y representación gráfica exigidos en la Decisión Andina 486 de 2000.

En el derecho comparado, si bien al igual que en Colombia no hay un consenso sobre la registrabilidad de los signos olfativos como marcas, se han planteado alternativas para poder obtener el registro de marcas olfativas.

Una de estas alternativas, es utilizar las herramientas disponibles en el marco del derecho de marcas, como lo sería la teoría del secondary meaning aplicado a los signos olfativos, con la finalidad de demostrar la fuerza distintiva de los olores. Otra de las alternativas para poder acceder al registro de los signos compuestos por olores es la flexibilización del requisito de representación gráfica con la finalidad de aceptar la descripción del olor por medio de palabras.

En algunas legislaciones, por la presión de estudiosos y las necesidades del mercado, se ha optado por la eliminación del requisito de representación gráfica del ordenamiento jurídico con el objetivo de facilitar el registro de los signos no tradicionales, entre ellos las marcas olfativas.

En Colombia, es menester mencionar que, a pesar de no se ha logrado el primer registro de un olor como marca, no es imposible lograrlo, ya que en las solicitudes de registro de marca no se han utilizado todos los métodos tecnológicos y legales disponibles para lograr este objetivo, más aun teniendo en cuenta la inexistencia de interpretaciones jurisprudenciales sobre la registrabilidad de los signos olfativos.

Finalmente, la evolución de la tecnología puede resolver los debates acerca de la posibilidad de proteger los aromas u olores como marcas en el sentido de que los empresarios puedan encontrar formas de preservar los olores sin que estos sufran alteraciones por los cambios climáticos o el paso del tiempo, o se pueden representar de forma visual, de esta forma se satisfacen de forma más simple los requisitos exigidos en la ley. 


\section{REFERENCIAS}

Arias, J. J. (2015). La registrabilidad de las marcas no tradicionales. Bogotá, D.C.: Superintendencia de Industria y Comercio.

Balañá, S. (2006). El entorno digital ¿segunda oportunidad para la marca olfativa? Estudio acerca de la capacidad del signo olfativo para funcionar como marca en el mercado. Actas de Derecho Industrial, XXVI (26), 19-57.

Cárdenas, P. E. (2003). Comentarios sobre propiedad intelectual. Bogotá, Colombia: Ediciones Pablo Emilio Cárdenas Pérez.

Castro, J. D. (2012). Las marcas no tradicionales. Revista La Propiedad Inmaterial (16), 297-325.

Castonguay, S. (2009). Olfato, oído, gusto - Los sentidos de las marcas no tradicionales. Revista de la OMPI (1), 4-6.

De Vega, M. (2001). Introducción a la psicología cognitiva. Madrid, España: Alianza Editorial.

Díaz, M. C y Pérez, M. S. (2006). Análisis de los compuestos responsables del aroma de las especias. Anuales de la Real Sociedad Española de Química 102(3), 31-35.

Escoffie, L y Jin, A. (2003). Exhalar o no una fragancia, esa es la cuestión: Un análisis comparativo de las marcas olfativas en la Unión Europea y los Estados Unidos de América como buenas oportunidades de comercialización para las Pymes. Disponible en http://www.wipo.int/export/sites/www/sme/ es/documents/pdf/olfactive_trademarks.pdf

Fernández-Nóvoa, C. (2004). Tratado sobre marcas. España: Marcial Pons.

Hernández, M. (2008). Los nuevos productos y las marcas olfativas. Anuario Facultad de Derecho - Universidad de Alcalá (21), 139-163.

Kresalija, B. (2001). La registrabilidad de las marcas auditivas, olfativas y las constituidas por color único en la decisión 486 de la Comunidad Andina de Naciones. Themis (42), 163-184.

López, L. y Ramón, J. (2017). ¿Qué sabemos de? El olfato. España: Consejo Superior de Investigaciones Científicas.

Llogrebat, M. (2007). Temas de Propiedad Intelectual. Alicante: La ley.

Martin, J. y Zuccherino, D. (2010). Cartas y comentario, primer registro de una marca olfativa en Argentina. Revista de la OMPI (2), 28. 
Martínez, J y Vargas, I. (2015). La afectación de la marca de tabaco por las medidas de empaquetado genérico, Vniversitas, 64(130), 235-272.

Metke, R. (2006) Lecciones de propiedad industrial III. Bogotá, Colombia: Baker \& Mackenzie.

Montoya, R. (2014). Manual de propiedad industrial. Bogotá, Colombia: Legis.

Organización Mundial de la Propiedad Intelectual (OMPI). (2006). Nuevos tipos de marcas. Comité Permanente sobre el derecho de Marcas, Diseños Industriales e Indicaciones Geográficas. Decimosexta sesión Ginebra, 13 a 17 de noviembre de 2006. Ginebra, Suiza.

Otamendi, J. (1999). Derecho de marcas. Buenos Aires, Argentina: Abeledo Perrot.

Pachón, M. y Sánchez, Z. (1995). El régimen andino de la propiedad industrial. Bogotá, Colombia: Ediciones Jurídicas Gustavo Ibáñez.

Parra, C. (2001). Distintividad, vulgarización y segundo significado de las marcas en la Decisión 486 de la Comisión de la Comunidad Andina. Revista La propiedad intelectual (3), 3148.

República de Colombia. Superintendencia de Industria y Comercio. (2005). Expediente 05047635. Recuperado de http://sipi.sic.gov. co/sipi/Common/Utils/GetFile.aspx?\&id=09000002803ca341

Sauri, S. (2014). Marketingdencias. Curiosidades y anécdotas sobre el marketing y la publicidad de tu día a día. Barcelona, España: Centro de libros PAPF, S.L.U.

Tribunal de Justicia de la Comunidad Andina. (18 de abril de 2012). Proceso 26-IP-2012.

Tribunal de Justicia de la Comunidad Andina. (20 de febrero de 2014). Proceso 240-IP-2013 [MP: Luis José Diez Canseco Núñez].

Tribunal de Justicia de la Comunidad Andina. (15 de marzo de 2013). Proceso 08-IP-2013.

Tribunal de Justicia de la Comunidad Andina (24 de agosto de 2015). Proceso 242-IP-2015. [MP: Luis José Diez Canseco Núñez].

Tribunal de Justicia de la Comunidades Europeas. (9 de diciembre de 2002). Asunto C-273/00. Caso Ralf Sieckmann/Deutches Patent-und Markenamt. [MP: F. Macken]. 